\title{
FUNDAMENTAL ASPECTS OF RADIO COMMUNICATION
}

A JOINT meeting of the Institute of Radio A Engineers and the American Section of the International Scientific Radio Union was held at Washington, D.C., on April 26, 1940. Fifteen papers on the more fundamental aspects of radio communication were presented.

H. T. Stetson, of the Massachusetts Institute of Technology, Cambridge, Mass., reported on a comparison of field strength measurements with auroral occurrences and ionospheric disturbances during 1930-40. His results indicated that, beginning six days before the date of the aurora, field strengths of $770 \mathrm{kc} . / \mathrm{s}$. were abnormally high with a maximum intensity occurring on the average four days before the date of the occurrence of the aurora. From three days before until two days after the auroral occurrence field strengths decreased to a minimum and remainèd abnormally low until about six days after. For higher frequencies involving the $F$ layers the auroral occurrences were compared with the transmission disturbance figures of the Bell Telephone Laboratories. Transmission disturbance was a minimum four days before the auroral occurrence, rose rapidly to a maximum one-half day following the dates of auroras, and then subsided to near the average value six days after the dates of the auroras. These results led to the conclusion that maximum transmission disturbance follows auroral phenomena and that the $F$ layers are affected on the average about one day earlier than the $E$ layer, based on more than three hundred days of observations utilized.

J. Bartels of the Carnegie Institution of Washington, N. H. Heck of the United States Coast and Geodetic Survey, and H. F. Johnston of the Carnegie Institution of Washington, all of Washington, D.C., described a new measure of geomagnetic activity, the three-hour-range index $K$. Each collaborating magnetic observatory assigns to each of the eight three-hour intervals of the Greenwich day one of the integers $0-9$ as a range index $K$, by a method which effectively separates the two main solar influences on the ionosphere, namely, $P$ (supposedly due to particles, and strongest in polar regions) and $W$ (supposedly due to wave-radiation, restricted to the daylight hemisphere). It was described how $P$ was measured by $K$, and a scheme for a geomagnetic record of $W$ was sketched.

J. H. Dellinger and N. Smith of the National Bureau of Standards discussed the reliability of predictions of ionospheric characteristics and radio transmission. Starting more than a year ago, pre- dicted values of critical frequencies and maximum usable frequencies were published monthly for the month following that of publication, in the Proceedings of the Institute of Radio Engineers. These published predictions were compared with the values afterwards measured. The results showed that the expected accuracy originally stated, 15 per cent, had been well met. The limit of accuracy is determined by the minor unpredictable variations of solar activity from its trend in the 11-year cycle.

L. V. Berkner and S. L. Seaton of the Carnegie Institution of Washington reported some phenomena in the $\mathrm{F}_{2}$ region during geomagnetic disturbance. The ionospheric effects of ionosphere storms at an equatorial station were found to be similar to the well-known effects in temperate latitudes at night, but in the daytime the critical frequencies increased during a storm instead of decreasing, as they usually do in a temperate latitude.

In a paper by Messrs. Gilliland and Taylor, of the National Bureau of Standards, results were reported on ionosphere measurements during the eclipse of April 7, 1940. The recombination coefficient in the $E$ layer was found to be approximately $10^{-8}$ and that in the $F_{2}$ layer less than $10^{-10}$.

Olof E. H. Rydbeck of Harvard University showed that from a wave-mechanical interpretation of the propagation of electromagnetic waves in an ionized medium it was possible to calculate the true height of reflection from the virtual-heightfrequency records. Thus the actual electron distribution could be obtained from experimental data.

K. A. MacKinnon of the Canadian Broadcasting Corporation, Montreal, Canada, reported some field intensity measurements of 540-kilocycle groundwave propagation over the high-conductivity prairie provinces. The ground conductivities approached $10^{-12}$ electromagnetic units with a maximum near south central Saskatchewan. In certain directions the earth was electrically uniform to distances of about 400 miles.

I. E. Mouromtseff of the Westinghouse Electric and Manufacturing Co., Bloomfield, N.J., discussed forced-air versus water-cooling of large vacuum tubes. Mechanical and thermal limitations of air cooling devices were described. General rules for designing an air cooler were described.

Prof. E. L. Chaffee of Harvard University discussed space-charge relations in triodes and the characteristic surface of large vacuum tubes. It 
was shown theoretically and from experiments that the plate, grid, and total currents, in the absence of secondary emission, varied as the $3 / 2$ power of the plate voltage along lines of constant $L=e_{g o} / e_{p o}$, where $e_{g o}$ and $e_{p o}$ were measured from a displaced origin. The three currents were then expressed in the form $i=A e_{p}{ }^{3 / 2}(1+\mu L)^{3 / 2} F(L)$. The entire system of static curves for each current can be expressed by a single curve. A simplification in the experimental determination of the static curves was suggested, permitting the static curves to be plotted from a few measurements at low power. The effects of secondary emission were discussed and curves were given which would aid in the design of tubes in which secondary emission from the plate was suppressed.

Prof. W. G. Cady of Wesleyan University, Middletown, Conn., reported a new method for the determination of the axes of quartz crystals by means of etch figures. A. de Gramont has shown that when a beam of light is passed through a quartz-crystal slab, one face of which has been etched with hydrofluoric acid, the rays are refracted in different directions, so that a lens placed close to the crystal projects on to a screen an' image that is characteristic of the particular face that has been etched. If the etched surface is normal to the optic axis ( $Z$-axis) the pattern takes the form of a three-pointed star, the points indicating the direction of the $X$-axis with a precision of about a degree. In repeating Gramont's experiment the author found that the pattern could be viewed directly through a powerful lens, for example, a $\frac{1}{4}$-in. microscope objective. The method furnished for the first time a simple and accurate optical method for finding the direction of the electric axis on a $Z$-cut slab of quartz.

Prof. Karl S. Van Dyke of Wesleyan University, Middletown, Conn., discussed the use of an etched sphere of quartz in identifying the orientation of quartz plates. He pointed out some discrepancies and contradictions in published papers, particularly as they related to the distinction between right and left quartz and the temperature coefficient of various 'cuts' of quartz resonators. This paper aimed at rectifying the discrepancies and contradictions which appeared in and caused confusion in the literature. Numerous papers involving discrepancies were specifically cited. All the factors concerned, etch figures, optical rotation, electrical polarization, and resonant frequencies, were in entire agreement with the elastic constants given by Voigt, and his equations for rotating the axes of reference, when used with his conventions as to axes and signs of angles. Taken together they made it possible to determine the peculiar and sometimes unstated conventions used by others in their published data.
C. R. Englund, A. B. Crawford, and W. W. Mumford of Bell Telephone Laboratories, Holmdel, N.J., discussed the diurnal variation of ultra-highfrequency optical-path transmission. Continuous. records of ultra-high-frequency transmission on frequencies of 75 and 150 megacycles per second over a good 'optical' path showed variations in the received field. These variations were explained as being caused by wave interference, an interference which varied with the changes in the composition of the troposphere. The diurnal meteorological factors which affected the transmission were discussed.

Andrew Alford of Mackay Radio and Telegraph Co., New York, N.Y., and Sidney Pickles, International Telephone Development Co., New York, N.Y., described an ultra-high-frequency voltmeter of new design. This was made up of a currentindicating instrument associated with a closed quarter wave-length of line. The quarter wavelength of line was adjustable for a range of frequencies. The instrument was calibrated and held calibrations within the limits of engineering accuracy. Voltages instead of currents were then used with calculated surge impedances to measure transmitted power.

R. W. George of R.C.A. Communications, Inc., Riverhead, L.I., N.Y., reported on field intensity of motor-car ignition between 40 and 450 megacycles per second. The average field intensity varied about 2 to 1 over the frequency range. Vertical and horizontal polarization were compared and showed slightly greater field intensity for vertical polarization. New cars, old cars, and trucks showed no large differences of ignition field intensity.

Andrew Alford of Mackay Radio and Telegraph Co., New York, N.Y., discussed currents induced in wires by high-frequency electromagnetic fields. The experiments and theory showed that, except in special cases when resonance phenomena predominated, the induced currents were not even approximately sinusoidally distributed and that, therefore, theories based on the a priori assumed sinusoidal distributions were limited to wires of certain special resonant wave-lengths.

During the morning of April 27 an ionosphere conference of about forty persons was held at the Department of Terrestrial Magnetism, Carnegie Institution of Washington. The principal subjects discussed informally were : results of ionospheric observations during the solar eclipse of April 7, 1940 ; effects of magnetic storms on transmission conditions ; methods for determining true heights of ionosphere layers ; methods for predicting magnetic and ionospheric conditions; measures of radio transmission disturbance; the new rangeindex for measuring magnetic activity; present status of the Lorentz polarization correction. 